\title{
Authors' reply: Prioritisation of infectious diseases in public health: feedback on the prioritisation methodology, 15 July 2008 to 15 January 2009
}

A Gilsdorf (GildorfA@rki.de) ${ }^{1}$, G Krause ${ }^{1}$

1. Department for Infectious Disease Epidemiology, Robert Koch Institute, Berlin, Germany

Citation style for this article:

Gilsdorf A, Krause G. Authors' reply: Prioritisation of infectious diseases in public health: feedback on the prioritisation methodology, 15 July 2008 to 15 January 2009. Euro Surveill. 2011;16(27):pii=19912. Available online: http://www.eurosurveillance.org/ViewArticle.aspx?Articleld=19912

Article published on 7 July 2011

To the editors: We thank the authors of the letter in reply to our article „Prioritisation of infectious diseases in public health: feedback on the prioritisation methodology, 15 July to 15 January 2009 “ for contributing to the discussion, that we initiated by launching the described feedback survey on the prioritisation methodology.

The points raised are mainly addressing concerns against the original prioritisation method, described in „Prioritisation of infectious disease in public health - call for comments“ by Krause et al. in 2008. As mentioned in our article in 2011, the survey was launched in order to get outside expertise for improving the prioritisation methodology, as we were preparing a new round of prioritisation. The suggestions that the survey participants raised were included in our review of the method. As the authors of the letter repeat several of the concerns addressed by the participants, it should be pointed out that they were taken into consideration in the latest prioritisation.

We are aware that some of the criteria are not exclusive and interdependent on each other. That was partly changed in the new round, but as we consider the criteria „incidence“, as a very relevant criteria, we decided to score some other criteria based on their effect on the population and not the individual, taking incidence again in account. The majority of participants considered a three tiered criteria scoring as sufficient, and it is challenging enough to define three scores for each criteria and often estimation is needed for the scoring. The use of a 0-100 scale would suggest a precision, that is often not reflected in reality. As mentioned in our article, there was ambiguity in some score descriptions. In the new round we tried to give clearer guidance on how to score in this situation. We were also aware that the categorical scoring of weights was not optimal and changed that in the new round. And we have defined a five year time period for the recent prioritisation, acknowledging the need for such a time frame.
We are pleased about the attention regarding prioritisation in public health, and that many of the concerns of the authors of the letter were reflected in the reply of the survey participants. These concerns are therefore addressed in the revised prioritisation method. This method was used for the latest prioritisation round, that was finished in February 2011 and is in the review process for publication right now. As we believe that such prioritisation has to be updated regularly, we are looking forward to continue the discussion and development of the methodology also in the future. 